came into view when, at the consultation, in response to insistent requests, the clothing was removed from the chest. The clue to the nature of the pleural effusion was evident. The possibility that a scar may mean the previous removal of a malignant growth should always be taken into consideration, whether such scar be on the lip, the scrotum; cervix uteri, the breast-in fact, anywhere. An absent eyeball has more than once led to the diagnosis of melanotic sarcoma of the liver.

In conclusion it may not be amiss, after having referred so often to the necessity of a thorough examination of these patients in whom there are obscure postoperative manifestations of malignant tumors, to call attention to the importance of such investigation before operation. A thorough exploration of the pelvis per vaginam and per rectum would sometimes ward off the useless operation for carcinoma of the stomach or breast. Secondary peritoneal tumors, as is well known, may sometimes plainly be made out on rectal examination. Such examination may readily be omitted when there are no pelvic symptoms, and when a palpable tumor of the stomach invites to operation. Unless obstructive or other urgent indications point to removal of the primary growth the detection of secondary deposits will contraindicate the operation. In three cases of hypernephroma I have seen operative removal of the kidney vetoed, once because of a secondary tumor in the brain, another time because of secondary deposits in the lung, and in a third case, the deciding voice being that of the late Christian Fenger, because edema of the leg made growth into the vena cava probable, and autopsy showed this to be the case. In a recent case secondary tumors in the lung decided against removal of a carcinomatous tongue. Percussion, auscultation, and the Roentgen rays all clearly showed the invasion of the chest. Such a warning as this may seem uncalled for. But clinical experience has made me feel that it may not be out of place to speak of these things, for it is often the well-known and commonplace that is forgotten, and that from time to time needs to be brought back to our attention.

\title{
VALUE OF RADIUM, SUPPLEMENTED BY CROSS-FIRE ROENTGEN RAYS, IN TREATMENT OF MALIGNANCY. ${ }^{1}$
}

\author{
By Russell H. Boggs, M.D.,
}

ROENTGENOLOGIST, ALLGGHENY GENERAL HOSPITAL; DERMATOLOGIST AND ROENTGENologist, PITTSBURGH, COLUMBIA, AND ST. FRANCIS HOSPITALs, pittseufgh, pennsylvania.

MucH discussion has taken place in regard to the comparative value of radium, mesothorium, and the Roentgen rays in the

' Read before the Allegheny County Medical Society, January 19, 1915. 
treatment of malignancy, but in view of the present knowledge of radio-active substances and the Roentgen rays, it is impossible to advocate the extended use of one to the exclusion of the other. Each agent has its place. Both forms of radiation have wide ranges of usefulness which differ under certain conditions and in adaptability to parts affected. When the gamma rays of radium are filtered from the alpha and beta it is found that they conformed in most respects to that of the Roentgen rays. During the past few years physicists have proved that both the Roentgen rays and the gamma rays are ether impulses identical in nature, differing only in wave length and power of penetration.

While today we are using Roentgen rays of much greater penetrating power and filtering out the lower inefficient rays, we must use different apparatus before we can produce rays with as great penetrating power as the highest gamma rays of radio-active substances. Many claim that the highest rays are unnecessary and have no therapeutic effect. This is a question still unsettled. Dessauer, of Frankfort, Germany, has constructed an apparatus which he claims produces rays which are, by every physical and biological test, identical with the gamma rays. He was to demonstrate this at the last meeting of the American Roentgen Ray Society in Cleveland, but, on account of the European War, was unable to be present.

The new tube invented by Coolidge, and used in many laboratories during the past year, marks a great advance because it provides much more uniform radiation which is essential in deep therapy. Coolidge is experimenting with a ray of much greater penetrating power than anything known except that produced by Dessauer, but has not placed this tube on the market. In a letter which I received from him recently he stated he would soon have this tube perfected by which any degree of penetration can be obtained.

With our present apparatus the therapeutic effects with the highest filtered Roentgen rays closely approach those of radium. There is no question that heavy doses of the filtered rays extending over not too long a period will cause the healing or disappearances of many malignant conditions which were unaffected by the inefficient treatment given a few years ago. After selecting the case, success or failure is rather a matter of technique, whether using radium or the Roentgen rays.

Both radium and the Roentgen rays as they are now understood have a different place in medicine and surgery than they had a few years ago. Those using radium had only small quantities, and often it was not of good quality, and the operators knew little or nothing about the necessity of filtering out the soft rays which did not penetrate, but which really predominated and acted as a caustic on the superficial tissues, nor did they understand much 
about secondary radiation. The same was true with those using the Roentgen rays. Any kind of a vacuum tube was used, no filters were employed, and usually the rays were directed in one direction toward the growth instead of using the present crossfiring methods, and with the tube placed at any distance regardless of the intensity without any instruments being used to measure the dosage.

There has been some rivalry between those using radium and the Roentgen rays, and as fast as one progressed the efforts of the other party to overtake its rival became more active. The inefficient Roentgen-ray work done in the early days and that which is being done at the present time by many accounts for some of the radium advocates making statements that there is no comparison in results between those produced by radium and the Roentgen rays. Many radium enthusiasts who are just beginning are making extravagant claims for radioactive substances because they have seen a few brilliant results for the first time, and they might be compared to the early Roentgen-ray extremists. Radium is an extremely valuable agent in the radiotherapeutic field, and extravagant and unwarranted claims will do much to hinder the avdancement of this branch of medicine. It can readily be seen that many physicians are judging both agents wrongly by the work being done by unqualified operators.

The experienced radiotherapeutists are the ones to whom we must still look for true information, rather than in books and the many periodicals lately published. The field of radiotherapy, meaning treatment by both radium and the Roentgen rays, is at present a kind of no one's ground and is suffering from incursions from every province of medicine and owning definite allegiance to none.

In treating a case either by radium, mesothorium, or the Roentgen rays we must always face a series of problems. Given a case with a certain lesion, its position, extent, its susceptibility to the influence to this or that radiation, when we must determine the agent or agents to use. The duration and method of application can be varied almost to infinity. .This enables us to realize how rich radiotherapy should be in its results when properly selected and employed. In this connection let me quote several paragraphs from Dr. Francis Hernaman-Johnson in the October number of the Archives of the Roentgen Ray:

"It is, at any rate, certain that in cancer the therapeutic effects of hard filtered Roentgen rays closely approach those of radium. On the other hand, a wide gap yawns between the Roentgen-ray results of today and even those of a few years ago. A claim often set forth in papers and books on radium therapy is that rodent ulcers have been cured by radium after months of Roentgen-ray treatment had proved ineffective. My own experience would 
lead me to believe that sharp doses of filtered Roentgen rays extended over a period not exceeding one month will cause the healing of any rodent ulcer which is amenable to radioactive substance in any form. . . . The employment of rays which are too soft, or those of insufficient doses extending over a long period, does positive harm. If, however, the mischief is not too far advanced a few large applications of filtered Roentgen rays may cause healing, just as effectively as gamma rays."

In speaking of postoperative treatment Dr. Hernaman-Johnson describes the form of radiation in the following words: "An approximately uniform dosage throughout the whole of the suspected area is, therefore, the ideal to be aimed at. For the complete realization of this ideal two things are necessary: (1) rays so penetrating that they will pass right through the body with the loss of only a small fraction of their value, and (2) a parallel beam of such rays so that they shall not lose energy by spreading. Gamma rays fulfil the first condition. They will pass through $60 \mathrm{~cm}$. of tissue before losing half their original intensity; but, to satisfy the second requirement, it would be necessary to suspend the tube some twenty inches from the skin, a procedure which would render useless even so large a quantity as $1000 \mathrm{mg}$. of radium salt. Hard Roentgen rays, on the other hand, though they fall to half value when they have penetrated about $10 \mathrm{~cm}$. into the body, can be produced in any desired amount, so that what is to all intents and purposes a parallel beam may be played upon selected areas. Existing Roentgen therapy thus approaches much more nearly the requirements of prophylaxis than treatment by radium."

Then Dr. Hernaman-Johnson continues: "One of the great advantages claimed for radium over Roentgen therapy is that a tube of the radioactive substance may be buried within a cancerous tumor. In accordance with the law of inverse squares, the activity of such a tube diminishes rapidly toward the outer parts of the growth; so that the risk, on the one hand, of not killing outlying cancer cells, and, on the other, of seriously injuring healthy tissues, is one to be very carefully considered.

A study of the literature of radium therapy in cancer shows that both these happenings are not remote contingencies, but have actually, in numerous cases, been the cause of failure. . If all tumors were perfect spheres it might be possible to calculate the exact quantity of radium required; but, unfortunately, cancer masses fail to conform to any of the figures with which we are familiar in text-books of plane and solid geometry. The recently introduced treatment by radioactive needles is open to the same objections, though in less degree. The only safe method, as in uterine cancer is to avoid huge doses, and to supplement the action of the radium tube by Roentgen-ray cross-fire outside."

Many observers who have used both agents extensively agree 
with Dr. Hernaman-Johnson, whose conclusions quoted are so clearly stated.

Every radiotherapeutist knows that the beam of rays given off from a Roentgen bulb or a radium tube is a mixture of heterogeneous rays, and that it is only by filtering and increasing the distance between the source of radiation that we can approach anything like a homogeneous ray. Then if we have homogeneous radiation we must not neglect the diminution of the distal dose by absorption by the tissues. There is always a difference between the proximal and distal dose. In using proper filtered radiation it has been estimated that each centimeter of tissue absorbs from 5 to 10 per cent. of radiation; so it can be readily seen that the deeper the growth is situated the more cross-firing with any form of radiation is necessary.

Dessauer considers that it would be necessary to have a radium tube containing 5 grams of radium when properly filtered and placed at the proper distance to give off a homogeneous ray equal to a bulb placed at the proper distance and properly filtered. No one has this amount or is it obtainable. This explains why most of the European workers who have had the best results in the treatment of malignancy long ago realized the importance of using the Roentgen rays from outside as an adjunct, and administering it through as many ports of entry as possible. In many places in the treatment of uterine cancer they used over forty ports of entry. This is a radical change from the technique that was used when the first cases of uterine cancer were treated by Roentgenotherapy years ago when little more than superficial or skin effect were produced. The treatment was given with an unshielded tube placed anteriorly to the abdomen the same as when making a radiogram.

It is generally accepted that equally good results can be secured by the use of radium or the Roentgen rays in the treatment of external epitheliomas, but, as a rule, most epitheliomas are treated by the Roentgen rays. It means nothing when some enthusiastic advocate of either the Roentgen rays or radium states that there is no doubt that one agent has been effective, whereas the other completely failed. Most likely the one applying the agent failed to recognize the limitations of his therapeutic agent, but when the epithelioma is situated on mucous membranes in cavities, radioactive substances will usually effect a cure unless too far advanced. Here radium always should have the preference. I believe no time should be lost in treating every case by radium as soon as diagnosed. The Roentgen rays should not be used through a speculum, thus losing valuable time if radium is available. I see so many patients coming late where the physician or surgeon had made an early diagnosis, and not knowing the value of radium, giving treatment by some other means when the lesion was situated so that it was inaccessible to any other form of treatment. Leukoplakia is a condition which can be cured by a few applications of radium or 
mesothorium and responds so unsatisfactory to any other therapeutic method. If left untreated the liability to ultimate malignant change must always be remembered. The stage at which an epithelioma situated on a mucous membrane is diagnosed and treated determines the prognosis more than any other malignant condition.

In carcinoma of the mouth, throat, rectum, or vagina the radioactive substance can be placed within the lumen of the organ or in close proximity to the growth, and, I believe, is superior to the Roentgen rays as far as the local treatment is concerned. But in all these cases-particularly if the disease is advanced and the lymphatics involved-the Roentgen rays are superior to any quantity of radium anyone has used up to the present time for the treatment of lymphatic glands. ' It must also be remembered that these high penetrating rays, given in great quantities and properly filtered, not only effect the adjacent lymphatic glands but also have a marked effect on the local tumor. In other words, it seems that the treatment is not complete if the radium is used locally unless it is followed for a certain length of time by Roentgenotherapy. Radiup might be compared to surgery in its action on the local tumor. The great advantage of the combined treatment is thus selfevident. Some inoperable cases of carcinoma which have not been cured have been improved to such a degree that a subsequent operation could be performed. No matter how rare these cases may be, every case should at least have this amount of palliation. It is certainly true that the diagnosis of an inoperable malignant growth should not be equivalent to a death warrant to the patient. Postoperative treatment carried out in this manner would undoubtedly increase the number of permanent cures. If radiotherapy could change the percentage of cures in only a small percentage of the cases it is more than justified. It would seem that this is not advising too much when some noted German gynecologists advise radiation as the only method of treatment of operable cases of cancer.

Besides using radium in cavities $I$ have been applying after operation a tube of radium in the axilla whenever it is possible, because it is often difficult or almost impossible in some cases for the patient to raise the arm sufficiently to give the desired amount of Roentgen treatment. Otherwise the results with modern Roentgen treatment are so good that, as far as external treatment is concerned, it seems radium has no advantage. When Roentgen rays are given prophylactically early, local recurrences are rarely seen. The great drawback is that the patients come to operation too late when deep metastasis has taken place. It is not enough in many cases to ray the axilla, the root of the neck, chest wall, and mediastinum, but the stomach and ovaries should receive attention. In order to have the best results the patients should not only come early but the lymphatics must receive radiation as uniformly as possible. 
In an article on "Carcinosis of the Bone, Secondary to a Growth in some Epithelial Organ."2 I mentioned the frequency of bone metastasis, and that it took place late, from three to five years after the cases were seen, and usually in the scirrhous type of carcinoma.

Limacher, who studied metastasis in mammary carcinoma, found the bones involved in about 14 per cent. of the cases. This shows the necessity of raying the cases thoroughly and early after operation, by the most efficient method possible.

In conclusion, the success of radium therapy in the treatment of malignancy is attained chiefly in those cases in which the radioactive substance is brought into contact with the growth, either in or on it, without an intervening layer of healthy tissue, and in which the thickness of the tumor does not exceed $4 \mathrm{~cm}$. It is preferable to use the hard Roentgen rays for all deep-seated growths in which there is an intervening layer of healthy tissue. Radium gives the best results when it is brought in contact with the growth and supplemented by the Roentgen rays from outside by the cross-fire method. It is necessary for the operator to know the relative value of radium and the Roentgen rays when combining these two agents.

\title{
BLOOD TRANSFUSION: INDICATIONS; RESULTS; GENERAL MANAGEMENT.1
}

\author{
By R. Ottenberg, M.D., \\ AND \\ E. Limman, M.D., \\ NEW YORK CITY.
}

(From the Mount Sinai Hospital, New York.)

BLoon transfusion, until recently, was expected to be a cureall, and was tried in almost every kind of desperate condition. As the result of a large amount of work done since Crile's introduction of a successful technic for direct blood transfusion, ${ }^{2}$ the real indications for transfusion have become better understood and more sharply defined. Crile's statement of the indications have, for the most part, remained valid, but subsequent

${ }^{2}$ New York Med. Jour., December 2, 1911.

${ }^{1}$ Various parts of this paper were presented at a stated meeting of the New York Academy of Medicine, December 17, 1914, and at the mceting of the Association of American Physiciaus, May 11, 1915.

${ }^{2}$ Canada Lancet, 1906-07, xl, 1057; Ann. Surg., 1907, xlvi, 329 , 